\title{
Performance Analysis of Exposed Terminal Effect in IEEE 802.11 Ad Hoc Networks in Finite Load Conditions
}

\author{
Dimitris Vassis and Georgios Kormentzas \\ Dept. of Information and Communication Systems Engineering, \\ University of the Aegean GR-83200, Karlovassi, Greece \\ \{Divas, gkorm\} @aegean.gr
}

\begin{abstract}
The paper evaluates the performance effects of exposed terminals in IEEE 802.11 ad hoc networks in finite load conditions. In this context, employing also models from previous authors' work, the paper derives analytical models for the estimation of channel utilization and media access delay for IEEE 802.11 ad hoc networks in finite load conditions with and without exposed terminals. The simulation results show that the analytical estimated channel utilization and media access delay metrics are fairly accurate.
\end{abstract}

\section{Introduction}

Due to the lack of a centralized control entity in ad hoc networks, sharing of wireless bandwidth among ad hoc terminals has to be organised in a decentralised manner. In this context, distributed Medium Access Control (MAC) mechanisms such as the IEEE 802.11 Distributed Coordination Function (DCF) [1], have gained widespread popularity in ad hoc networks. As all Carrier Sense Multiple Access with Collision Avoidance (CSMA/CA) based MAC protocols, DCF suffers from hidden and exposed terminal problems. The use of Request to Send/Clear to Send (RTS/CTS)-like schemes partially solves the hidden terminal problem, while it leaves the exposed terminal open, where some nodes that heard the RTS/CTS exchange refrain from transmission even though they would not have interfered with any going transmission, thus the result for the whole system is channel utilization and throughput reduction.

As the exposed terminal problem results in performance degradation of IEEE 802.11 ad hoc networks and DCF does not implement an appropriate mechanism to alleviate this problem, there are a lot of works at the international literature where the authors propose their own solutions by enhancing DCF (e.g., [2-4]). Independently of the various proposed solutions, the paper discusses the performance effects of exposed terminals in IEEE 802.11 ad hoc networks in finite load conditions. In this context, the paper derives appropriate fairly accurate analytical models for the estimation of channel utilization and media access delay for IEEE 802.11 ad hoc networks in finite load conditions with and without exposed terminals.

The rest of the paper is organised as follows. Section 2 gives a brief description of the DCF mechanism and presents the hidden and exposed terminal problems. Section 3 and 4 discus the proposed analytical utilization and delay models respectively, and Section 5 validates the accuracy of the analytical estimated metrics through appropriate simulation scenarios. Section 6 evaluates the performance effects of exposed 
terminals in IEEE 802.11 ad hoc networks and finally, Section 7, gives the concluding remarks of this work.

\section{Brief Description of the IEEE 802.11 DCF Mechanism}

According to DCF, a node can initiate a transmission only if it senses the medium as being idle for a time interval greater than a Distributed InterFrame Space (DIFS). If a collision occurs, the transmission is deferred and a backoff process starts. Unlike wired networks (with Carrier Sense Multiple Access with Collision Detection support), in a wireless environment, collision detection is not possible. Hence, an acknowledgement (ACK) frame is used to notify the sending node that the transmitted data has been successfully received. The transmission of ACK is initiated at a time interval equal to Short InterFrame Space (SIFS) that follows the reception of the sending data (see Figure 1(a)).

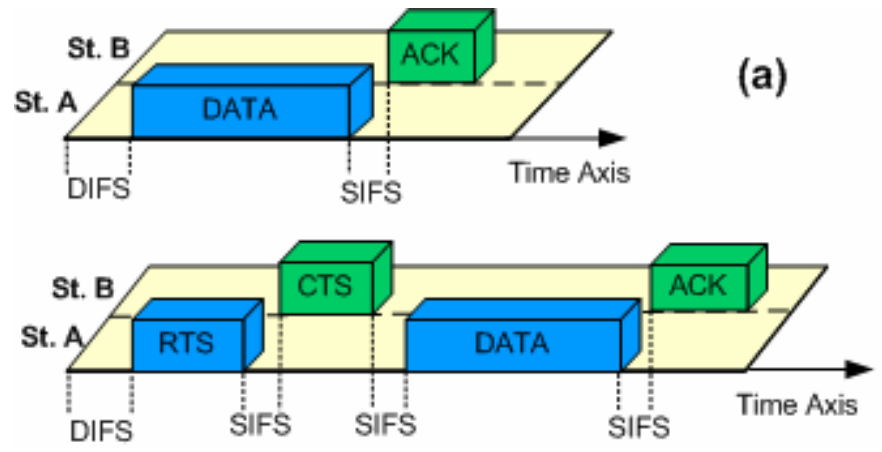

(b)

Fig. 1. Frame exchanges in a packet transmission procedure

The above transmission mechanism does not protect the wireless nodes from the hidden terminal problem (see Figure 2(a)). This problem arises, for example, when for
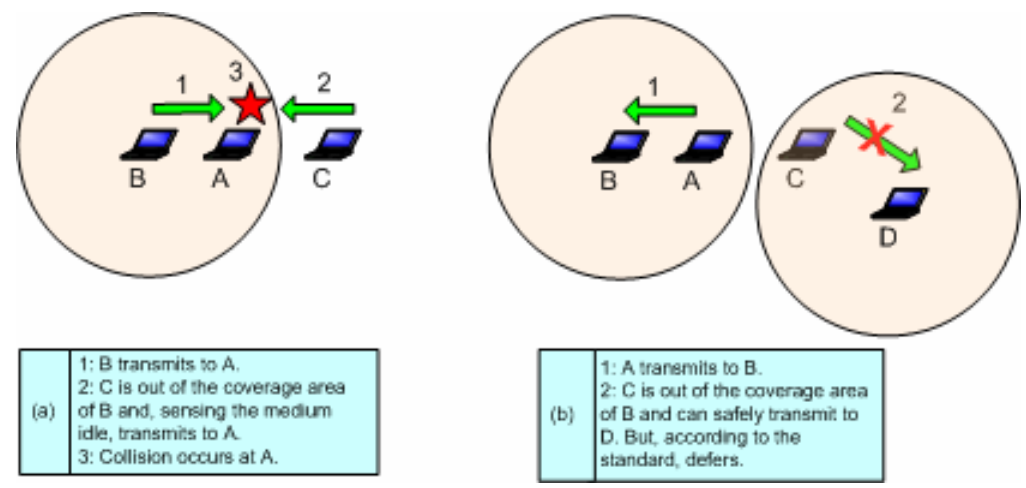

Fig. 2. The hidden and exposed terminal problems 
three stations, namely $\mathrm{A}, \mathrm{B}$ and $\mathrm{C}, \mathrm{A}$ is able to communicate with $\mathrm{B}$ and $\mathrm{C}$, but $\mathrm{C}$ is not within the range of $\mathrm{B}$. In this case, $\mathrm{C}$ is hidden for $\mathrm{B}$. This means that, when $\mathrm{B}$ transmits a frame to $\mathrm{A}, \mathrm{C}$ may sense the medium as being idle. If $\mathrm{C}$ also transmits a frame towards $\mathrm{A}$, then a collision will occur at $\mathrm{A}$.

In order to alleviate this problem, the IEEE 802.11 standard includes a virtual carrier sense mechanism, which is based on the exchange of two short control frames: a Request To Send frame (RTS), which is sent by a potential transmitter to the receiver and a Clear To Send frame (CTS), which is sent back from the receiver in response to RTS. The RTS and CTS frames include a duration field that specifies for a station the time interval necessary to completely transmit its data and the related ACK. Other stations can hear either the sender or the receiver and refrain from transmitting until the data transmission is complete (see Figure 1(b)). The RTS/CTS mechanism adds a considerable overhead in the medium, especially for the transmission of small data packets. In this context, the use of RTS/CTS is controlled through the RTS Threshold attribute, where only packets with size greater than the value of the RTS Threshold are transmitted with the RTS/CTS mechanism.

The exposed terminal problem is reverse to the hidden terminal one. Taking as reference Figure 2(b), A is able to communicate with B and C. C is able to communicate with $\mathrm{A}$ and $\mathrm{D}$ but it is outside the coverage area of $\mathrm{B}$. Now, during a frame transmission from A to B, C senses the medium busy and defers from transmitting a frame to $\mathrm{D}$, despite the fact that this transmission would not result in a collision, as $\mathrm{C}$ is outside the coverage area of $\mathrm{B}$, and $\mathrm{A}$ is outside the coverage area of $\mathrm{D}$. In this case $\mathrm{C}$ is exposed to A. Contrary to the hidden terminal problem, as already mentioned in the introductory section, the IEEE 802.11 standard does not include an appropriate mechanism to alleviate the exposed terminal problem.

\section{The Proposed Analytical Utilization Model}

This section presents an analytical model that estimates the channel utilization effect of exposed terminals in IEEE 802.11 ad hoc networks. In order to derive this estimation, the paper employs previous authors' work [5] concerning the analytical estimation of channel utilization for IEEE 802.11 ad hoc networks under the hidden terminal problem. Note that the channel utilization is considered to be the percentage of time in which useful information is transmitted in the wireless medium. Firstly, we give a brief overview of the model presented in [5] and then we proceed to estimate the channel utilization effect of the exposed terminals.

Figure 3 depicts a conventional IEEE 802.11 ad hoc network where the nodes' transmission range is $r$ and the wireless channel data rate is $R$. Let now consider the node A of Figure 3. The small circle includes the nodes that are inside the coverage area of node A. The big circle contains also the hidden nodes for node A, which are the nodes at the ring between the big and the small circles.

Our work in [5] derives the channel utilization in respect to the aggregate traffic produced in the small circle. [5] assumes that the aggregate offered traffic load in the 


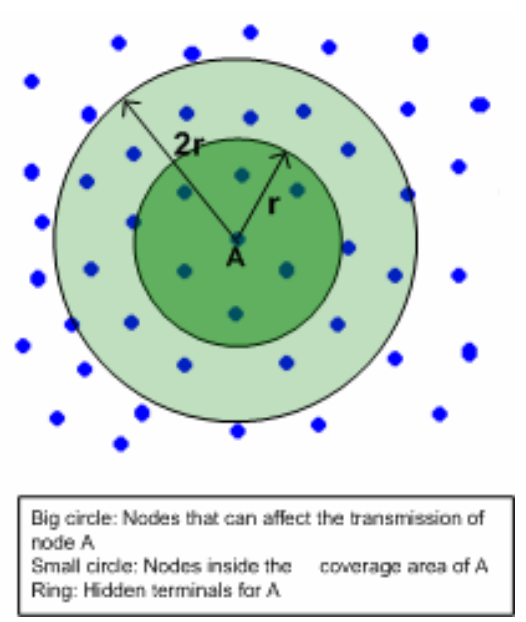

Fig. 3. A conventional IEEE 802.11 ad hoc network

wireless channel is generally distributed with a mean value of $g$ packets per second and that the payload size of packets transmitted in the wireless medium is also generally distributed with a mean value of $P$ bits. It also assumes that the network operates under finite load conditions, so, the offered traffic consists not only of new packets but also of previously collided packets.

According to [5], the channel utilization related to a random node of a conventional IEEE 802.11 ad hoc network is

$$
U=\frac{S}{(B+I)^{4}} I^{3} p^{3 T_{s} / \tau}
$$

where $S$ is the average transmission time of the packet payload in the wireless medium, $B$ is the average busy period of the medium, $I$ is on average the idle period of the medium, $\tau$ is the duration of an IEEE 802.11 time slot [1], $p$ is the probability that no packet arrives in a time slot, and $T_{s}$ is the time needed for a successful transmission.

Again according to [5],

$$
\begin{gathered}
I=\frac{\tau}{1-p}, \\
B=\frac{T_{c}+p_{s}\left(T_{s}-T_{c}\right)}{p}, \\
S=\frac{P p_{1}}{R p(1-p)},
\end{gathered}
$$




$$
p_{s}=\frac{p_{1}}{1-p}
$$

where $T_{c}$ is the time a collision takes (explicit expressions for $T_{s}$ and $T_{c}$ are given in [6]), and $p_{1}$ is the probability that a single arrival occurs in a time slot.

\subsection{Analysis of the Utilization Effect of the Exposed Terminals}

Let now take a look at Figure 4, where we consider that a transmission is in progress from node $\mathrm{A}$ to a random node inside the coverage area of $\mathrm{A}$, i.e., a circle with centre A, named $E$. We furthermore assume that this transmission does not refer to node B. According to the IEEE 802.11 standard, if node B has a packet to send, it has to wait until the transmission concerning $\mathrm{A}$ is completed.

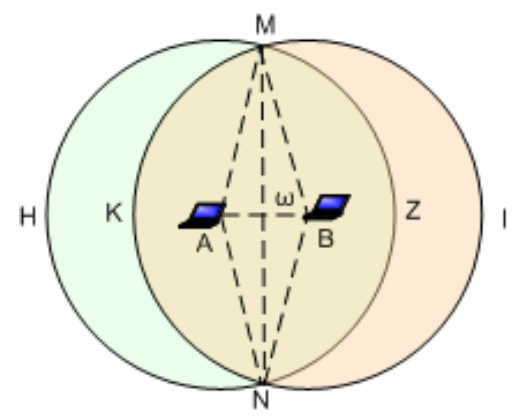

Fig. 4. Exposed terminal effect analysis

If the transmitted packet from $\mathrm{A}$ is destined to a node inside the area $E_{1}$ that is the area (NKMZ), then node B has to defer its transmission, as otherwise a collision will occur to the receiving node. However, if the transmitted packet from $\mathrm{A}$ is destined to a node inside the area $E_{2}$ that is the area (NHMK), then node B could not have to defer as the receiving node is outside its coverage area. But, still, in this second case, according to the standard, B has to defer. Hence, by assuming that nodes are uniformly distributed across the network area and transmissions occur equiprobably to all nodes, when node B wants to transmit and the medium is busy, it has to wait pointlessly in a fraction $E_{2} / E$ of its anticipated transmissions.

We proceed now to examine the effect of the above pointless deferrals in the network's utilization. Assume that the IEEE 802.11 standard includes a sophisticated solution in the exposed terminal problem, such that a node knows when to defer from transmission while another one transmits, and when not. The probability that a deferral occurs because the medium is busy is equal to the probability that the medium is busy, which is $B /(B+I)$, times the probability that a transmission occurs in a slot, which is $(1-p)$. If nodes know when to transmit during another transmission and 
when not, then a percentage $\beta=\mathrm{B} /(\mathrm{B}+\mathrm{I}) \cdot(1-p) \cdot\left(E_{2} / E\right)$ of additional transmissions will occur in the medium, simultaneously with transmissions that are already in progress. This means that we will have a virtual additional channel where transmissions occur with packet generation rate equal to $g_{e}$, such that the probability that a packet arrives in a time slot is $\beta$. Apparently, the parameter $g_{e}$ can be easily derived from $\beta$ if we know the distribution describing the packet generation rate.

The fraction $E_{2} / E$ is derived in Appendix equal to 0.42. Accordingly, the value of $\beta$ from which $g_{e}$ arises is

$$
\beta=\frac{0.42 \mathrm{~B}(1-p)}{B+I} .
$$

Eventually, the extra channel utilization if the exposed terminal problem would not exist can be derived from Equation 1 by replacing $g$ with $g_{e}$. Accordingly, the total channel utilization in the ideal absence of the exposed terminal problem is

$$
U=U(g)+U\left(g_{e}\right) .
$$

\section{The Proposed Analytical Delay Model}

This section discusses an analytical model that estimates the media access delay effect of exposed terminals in IEEE 802.11 ad hoc networks in finite load conditions. The media access delay is the time from the beginning of a packet transmission from a node till its successful reception from the next node.

For the media access delay estimation, consider again a node (e.g., node A of Figure 2) and a cell that includes all nodes in the coverage area of the considered node. Let $G$ be the normalized traffic of a cell with respect to the packet transmission time, given as $G=g \cdot P / R$. Moreover, the mean number of retransmissions before a packet is sent is $G / U$ [7]. Excluding the last, successful transmission, the actual mean number of retransmissions is $m=(G / U)-1$. Considering a large packet inter arrival time compared to the time slot, we can assume that the packet does not wait for a DIFS interval in the first transmission attempt. The expected media access delay of a packet transmitted from a node is equal to the duration $T_{s}$ needed for the packet to be successfully transmitted plus the time taken for all its unsuccessful transmissions. The second is equal to the expected duration of each unsuccessful transmission (backoff time plus the duration $T_{c}$ of a collision) times the number of unsuccessful transmissions, $m$. Accordingly, the media access delay is given as

$$
d_{m}=T_{s}+m\left(T_{c}+\tau \bar{X}+\bar{F}\right),
$$

where $\bar{X}$ is the expected number of backoff slots in a retransmission and $\bar{F}$ is the time where the backoff counter freezes because of transmissions that are in progress. 
Considering that the backoff slots in each attempt are uniformly distributed between 1 and the contention window, then

$$
\bar{X}=\frac{1}{m} \sum_{j=0}^{m-1} W 2^{j}=\frac{1}{2 m} W 2^{m-1},
$$

where $W$ is the minimum contention window.

For the time $\bar{F}$ that the backoff counter freezes because of another transmission, consider that the probability that the backoff counter expires is simply $1 / \bar{X}$. Under the finite load conditions of our case, the transmitter of a node can be thought as a G/G/1 queue with utilization factor $u=g_{N} d_{m}$, where $d_{m}$ is the media access delay and $g_{N}$ is the node traffic. The probability that a node transmits is equal to the probability that the backoff counter expires, given that it has a packet in the transmitter. This is $u / \bar{X}$. Hence, if $M$ is the number of nodes in a cell, in $\bar{X}$ slots where the backoff counter decreases, there will occur $M \cdot(U / \bar{X}) \cdot \bar{X}=M \cdot g_{N} \cdot d_{m}=$ $g \cdot d_{m}$ additional transmissions. From all these transmissions there are $g \cdot d_{m}$ $[m /(m+1)]$ retransmission and $g \cdot d_{m}[1 /(m+1)]$ successful transmissions. In this way all these transmissions add an additional backoff time of

$$
\bar{F}=g \cdot d_{m}\left[T_{s} /(m+1)+m T_{c}(m+1)\right] .
$$

Eventually, the media access delay can be derived by substituting $\bar{F}$ into Equation 8 , giving

$$
d_{m}=\frac{m\left(T_{c}+\tau \bar{X}\right)+T_{s}}{1-g m\left(T s+m T_{c}\right) /(m+1)}
$$

\subsection{Analysis of the Delay Effect of the Exposed Terminals}

For deriving the media access delay if the exposed terminal problem would not exist, we assume again that the IEEE 802. 11 standard includes a sophisticated algorithm where nodes know when to transmit and when not, during another transmission in progress. Following this line of thought, if a node freezes its backoff counter for $g \cdot d_{m}$ transmissions that occur from other nodes (as denoted above), then in the case where the exposed terminal problem is solved, it would only freeze the backoff counter for a fraction $\left(1-E_{2} / E\right)$ (that is 0.58$)$ of these transmissions only. Consequently, Equation 10 is rearranged as follows:

$$
d_{m}=\frac{m\left(T_{c}+\tau \bar{X}\right)+T_{s}}{1-0.58 g m\left(T s+m T_{c}\right) /(m+1)}
$$

In the above Equation, $m$ is derived through $U$ from Equation 7. 


\section{Models Validation}

In order to validate the accuracy of the derived in the previous sections analytic approximations of channel utilization and media access delay effects of exposed terminals in ad hoc networks performance, several simulation scenarios were considered in the Pythagor simulation platform [8], an open C++ simulation tool for IEEE 802.11 $\mathrm{a} / \mathrm{b} / \mathrm{g}$ networks.

The estimated models stand for any input traffic model, as long as the parameters $g, p, p_{1}$ and $P$ are given in a closed form. For our validation tests, although the input traffic depends on many characteristics specific to each network, the Poisson distribution with a mean value of $g$ packets/s was adopted to efficiently characterize the aggregated generated traffic inside a cell. Consequently, $p, p_{1}$, and $g_{e}$ (for the exposed terminal problem analysis) can be easily derived as follows:

$$
\begin{aligned}
& p=\frac{(g \tau)^{0}}{0 !} e^{-g \tau}=e^{-g \tau} \\
& p_{1}=\frac{(g \tau)^{0}}{0 !} e^{-g \tau}=g \tau e^{-g \tau} \\
& \beta=1-e^{-g_{e} \tau} \Rightarrow g_{e}=-\ln (1-\beta) / \tau
\end{aligned}
$$

In addition, an exponential distribution with a mean value of $P$ bits was chosen for the packet payload size. The exponential distribution is proven adequate to describe the packet size distribution in IEEE 802.11 networks (e.g., [6]).

Table 1 summarizes the input parameters used during the validation process.

Table 1. Simulation parameters

\begin{tabular}{|l|l|}
\hline Parameter & Value \\
\hline time slot duration $(\tau)$ & $20 \mu \mathrm{s}$ \\
SIFS & $10 \mu \mathrm{sec}$ \\
DIFS & $50 \mu \mathrm{sec}$ \\
minimum contention window $(W)$ & $31 \mathrm{slots}$ \\
PHY header & $96 \mathrm{bits}$ \\
MAC header & $272 \mathrm{bits}$ \\
ACK & $112 \mathrm{bits}+\mathrm{PHY}$ \\
RTS & $160 \mathrm{bits}+\mathrm{PHY}$ \\
CTS & $112 \mathrm{bits}+\mathrm{PHY}$ \\
Channel data rate $(R)$ & $5.5 \mathrm{Mb} / \mathrm{s}$ \\
RTS Threshold & $128 \mathrm{Bytes}$ \\
packet payload $(P)$ & $1 \mathrm{KByte}$ \\
\hline
\end{tabular}


Tables 2 and 3 summarize the results of the comparison between the analytical metrics and the Pythagor output. In all cases the results show that the models are fairly accurate.

Table 2. Model validation for the channel utilization effect of exposed terminals

\begin{tabular}{|c|c|c|c|}
\hline $\begin{array}{c}\text { Channel traffic }(\mathrm{g}) \\
\mathrm{Kb} / \mathrm{s} \\
(1 \text { packet }=8 P \text { bits })\end{array}$ & \begin{tabular}{|c|} 
Channel \\
utilization $(\%)$ \\
$($ Equation 7$)$ \\
\end{tabular} & \begin{tabular}{|c}
$\begin{array}{c}\text { Channel } \\
\text { utilization }(\%) \\
\text { (Pythagor) }\end{array}$ \\
\end{tabular} & $\begin{array}{c}\text { Difference } \\
(\%)\end{array}$ \\
\hline 1000 & 18.94 & 20.45 & 8 \\
\hline 2000 & 18.16 & 19.61 & 8 \\
\hline 3000 & 16.88 & 18.07 & 7 \\
\hline 4000 & 15.32 & 16.39 & 7 \\
\hline
\end{tabular}

Table 3. Model validation for the media access delay effect of exposed terminals

\begin{tabular}{|l|l|l|c|}
\hline $\begin{array}{c}\text { Channel traffic }(\mathbf{g}) \\
\mathbf{K b} / \mathbf{s}\end{array}$ & $\begin{array}{l}\text { Media access } \\
\text { delay (ms) } \\
(\mathbf{1} \text { packet }=\mathbf{8 P} \text { bits) }\end{array}$ & $\begin{array}{c}\text { Media access } \\
\text { delay (ms) } \\
(\text { Pythagor) }\end{array}$ & $\begin{array}{c}\text { Differ- } \\
\text { ence }(\boldsymbol{\%})\end{array}$ \\
\hline 1000 & 2.42 & 2.63 & 9 \\
\hline 2000 & 5.23 & 5.65 & 8 \\
\hline 3000 & 9.78 & 10.56 & 8 \\
\hline 4000 & 562.31 & 601.68 & 7 \\
\hline
\end{tabular}

\section{Performance Evaluation of Exposed Terminal Effect}

This section evaluates the utilization and delay effects of exposed terminals in an IEEE 802.11 ad hoc network that employs the parameters of Table 1. We concern three cases, where in the first case, we assume clear channel conditions, where all nodes are in line of sight with each other, in the second one, we assume real conditions, meaning existence of hidden and exposed terminals, and in the third one, we assume that the standard includes a sophisticated solution such that exposed terminals do not exist.

As it is shown in Figure 5 and obviously expected, the channel utilization for clear channel conditions is remarkably higher than the other two cases. Another important difference concerns the shapes of the three curves. This difference graphically depicts the effect of the exposed terminals. In the clear channel conditions case where both the hidden and the exposed terminal problems do not exist, most collisions are prevented through the RTS/CTS mechanism. As a result, even if the input traffic increases too much, the utilization does not decrease. In the real conditions case where both problems exist, hidden nodes transmit during the transmission of others and exposed nodes defer their transmissions pointlessly. Consequently, as the network traffic increases, so do the collisions caused from the aforementioned problems, resulting in rapid utilization impairment, which is depicted through the sharp shape of 


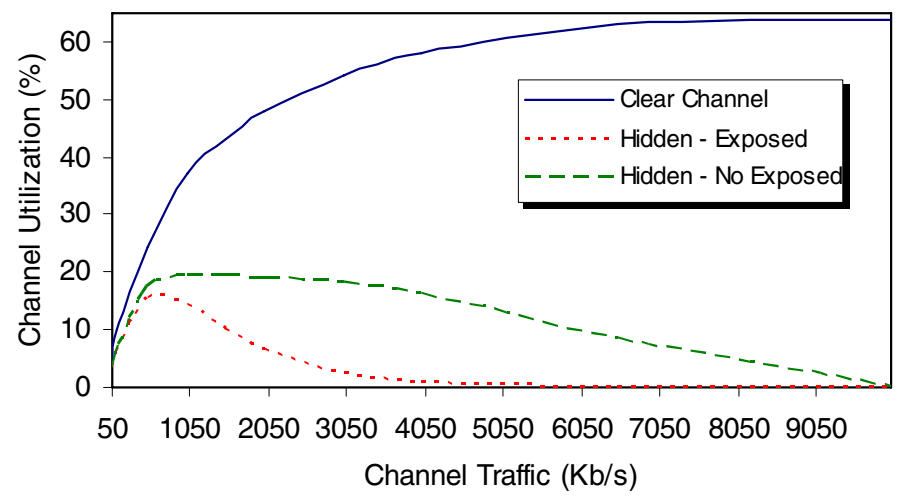

Fig. 5. Channel utilization effect of exposed terminals

the curve. In the case where the exposed terminal problem is treated, the collisions are less and the utilization impairment is slower and consequently the shape of the respective curve is smoother.

Figure 6 depicts the media access delay effect, which is totally aligned with the utilization one. The media access delay for clear channel conditions is very small. Moreover, it does not increase rapidly as in the other two cases. This is because the utilization does not decrease after a limit of channel input traffic, so the number of retransmission attempts increases at a rate less than linear. Furthermore, as it is expected the absence of exposed terminals keeps the media access delay remarkably lower than happens in real conditions case.

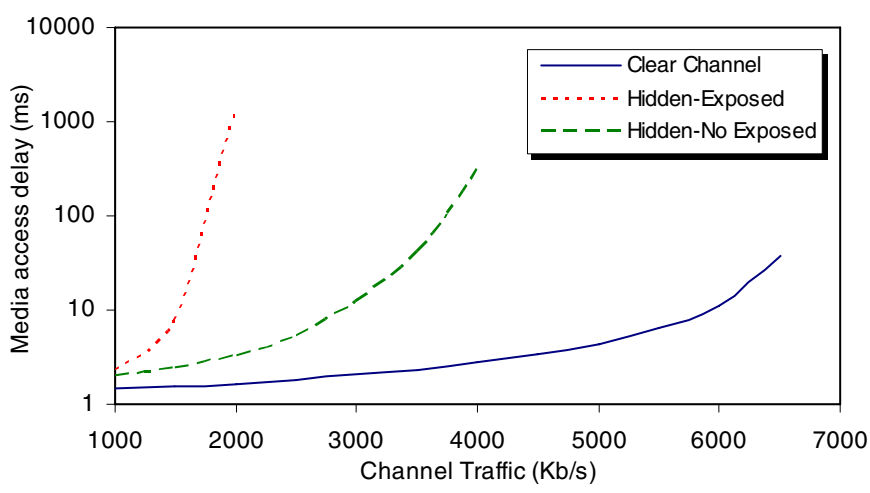

Fig. 6. Media access delay effect of exposed terminals

\section{Conclusions}

The paper presents analytical models for the estimation of channel utilization and media access delay effects of exposed terminals in IEEE 802.11 ad hoc networks in 
finite load conditions. In order to validate the accuracy of the analytic approximations, several simulation scenarios were considered in the Pythagor simulation platform. In all cases the results of the comparison between the analytical metrics estimate and the Pythagor output show that the models are fairly accurate.

\section{Acknowledgment}

The work reported in this paper is supported in part by 'Pythagoras II - Research Group Support of the University of the Aegean'.

\section{References}

[1] Wireless LAN Medium Access Control (MAC) and Physical Layer (PHY) Specification, IEEE Std. 802.11, 1999.

[2] F. Borgonovo, A. Capone, M. Cesana, L. Fratta, "ADHOC MAC: a new, flexible and reliable MAC Architecture for ad hoc Networks", In Proc. of 2003 IEEE Wireless Communications and Networking Conference, 16-20 March 2003, New Orleans LA, USA, Volume: 2, Page(s): 965-970.

[3] D. Shukla, L. Chandran-Wadia and S. Iyer, "Mitigating the Exposed Node Problem in IEEE 802.11 Ad Hoc Networks", In Proc. IEEE CCCNO3, October 2003, USA.

[4] A. Acharya, A. Mishra, and S. Bansal, "MACA-P: A MAC for Concurrent Transmissions in Multi-hop Wireless Networks", IBM Research Report RC22528, July 2002, In Proc. IEEE PerCom, 2003.

[5] D. Vassis and G. Kormentzas, "Throughput Analysis for IEEE 802.11 Ad Hoc Networks under the Hidden Terminal Problem"', In Proc. IEEE CCNC2006 HWN-RMQ Workshop, Nevada, USA, January 2006.

[6] G. Bianchi, "Performance Analysis of the IEEE 802.11 Distributed Coordination Function," IEEE Journal on Selected Areas of Communications, vol. 18, no. 3, pp. 535-547, 2000.

[7] L. Kleinrock and F. Tobagi, "Packet Switching in Radio Channels: Part I-Carrier Sense Multiple Access Models and their Throughput-Delay Characteristics," IEEE Transactions on Communications, vol. 23, no. 12, pp. 1400-1416, Dec. 1975.

[8] Pythagorsimulation tool, Available on line at the URL: http://www.icsd.aegean.gr/ telecom/pythagor/index.htm

\section{Appendix: Deriving the fraction E2/E}

As the fraction $E_{2} / E$ depends on the distance between the transmitting node (A) and the exposed node (B), we will derive it for the average distance between two nodes inside a cell. For two nodes $\mathrm{A}$ and $\mathrm{B}$, the probability that B is $x$ meters away from $\mathrm{A}$ is $2 \pi x d x / \pi r^{2}$. Consequently, the mean distance $\mathrm{d}$ is

$$
\bar{d}=\int_{0}^{r} \frac{2 \pi x}{\pi r^{2}} d x=\frac{2}{3} r
$$


Concerning Figure 4 and the notation used in Section 3, we need to derive the fraction $(M H N K) / \pi r^{2}$. For $\mathrm{d}=(\mathrm{AB})=2 \mathrm{r} / 3$, we have:

$$
\begin{aligned}
& (\mathrm{MHNK})=\pi \pi^{2}-(\mathrm{MKNZ}), \\
& (\mathrm{MKNZ})=2(\mathrm{MKN}), \\
& (\mathrm{MKN})=(\mathrm{BMKN})-(\mathrm{BMN}), \\
& (\mathrm{BMKN})=\frac{1}{2} r^{2}(2 \omega), \\
& (B M N)=\frac{1}{2} \frac{d}{2} 2 r \sin \omega=\frac{1}{2} d r \sin \omega .
\end{aligned}
$$

From the Cosines' Law in ABM, it is:

$$
r^{2}+d^{2}-2 r d \cos \omega=r^{2} \Rightarrow \omega=a \cos \left(\frac{d}{2 r}\right) .
$$

Combining the above we have

$$
(\mathrm{MHNK})=\pi r^{2}-2 \mathrm{r}^{2} \operatorname{acos}\left(\frac{\mathrm{d}}{2 \mathrm{r}}\right)+\operatorname{drsin}\left[\operatorname{acos}\left(\frac{\mathrm{d}}{2 \mathrm{r}}\right)\right] .
$$

For $\mathrm{d}=2 \mathrm{r} / 3$, the above equation becomes $\mathrm{E} 2=(\mathrm{MHNK})=1.3066 \mathrm{r}^{2}$, and the fraction $\mathrm{E} 2 / \mathrm{E}$ is

$$
E_{2} / E=\frac{1.3306 r^{2}}{\pi r^{2}}=0.42
$$

